\title{
AN ALGEBRA OF SINGULAR INTEGRAL OPERATORS WITH TWO SYMBOL HOMOMORPHISMS
}

\author{
BY H. O. CORDES ${ }^{1}$
}

Communicated by M. H. Protter, May 28, 1968

1. Let $R_{+}^{n+1}=\left\{(x, y)=\left(x_{1}, \cdots, x_{n}, y\right): x_{j}, y \in R, y \geqq 0\right\}$ and let $\Delta_{d}, \Delta_{n}$ denote the two unbounded positive self-adjoint operators of the Hilbert-space $\mathfrak{S}=\mathscr{L}^{2}\left(R_{+}^{n+1}\right)$ generated by closing the Laplace operator in $C_{0}^{\infty}\left(R_{+}^{n+1}\right)$ under Dirichlet and Neumann boundary conditions at $y=0$, respectively.

We propose to study the "convolution algebra" $\mathscr{L}^{\#}$ generated by the generalized Riesz-Hilbert-operators

$$
\begin{gathered}
\Lambda_{d}=\left(1-\Delta_{d}\right)^{-1 / 2}, \quad \Lambda_{n}=\left(1-\Delta_{n}\right)^{-1 / 2}, \quad S_{d}=-i \partial / \partial y \Lambda_{d}, \\
S_{n}=-i \partial / \partial y \Lambda_{n}, \quad S_{d, j}=-i \partial / \partial x_{j} \Lambda_{d}, \quad S_{n, j}=-i \partial / \partial x_{j} \Lambda_{n}, \\
j=1, \cdots, n
\end{gathered}
$$

and later on also will adjoin certain multiplications by continuous functions, to obtain an algebra $\mathfrak{A}$ of singular integral operators on the half-space $R_{+}^{n+1}$.

Both $C^{*}$-algebras $\mathfrak{R}^{\#}$ and $\mathfrak{A}$ have noncompact commutators, but each is commutative modulo a certain larger ideal (\$\# and \&, respectively). We therefore obtain a first symbol function $\sigma_{A}$ for $A \in \mathfrak{Q}^{*}$ (or $\mathfrak{A}$ ) which is a continuous complex-valued function over the maximal ideal space of $\mathfrak{H}^{\#} / \mathbb{E}^{\sharp}$ (or $\mathfrak{A} / \mathbb{E}$ ). If $\sigma_{A}$ does not vanish, we can invert the operator mod $\mathbb{E} \#$ (or $\mathbb{E}$ ), or reduce the singular integral equation $A_{n} u=f$ to an equation $(1+E) u=g$ with $E \in(5 ; 4$ (or (E).

Now, we find that the ideals $\&$ and $\&$ are isomorphic to topological tensor products of the form $\mathfrak{S}(\mathfrak{h}) \hat{\otimes} \mathfrak{S}^{\#}, \mathbb{E}=\mathfrak{C}(\mathfrak{h}) \hat{\otimes} \mathfrak{S}$, with respect to a suitable direct decomposition

$$
\mathfrak{G}=\mathfrak{h} \otimes \mathfrak{l}, \quad \mathfrak{h}=\mathfrak{L}^{2}\left(R^{+}\right), \quad \mathfrak{l}=\mathfrak{L}^{2}\left(R^{n}\right),
$$

where $\mathfrak{S}(\mathfrak{h})$ denotes the compact ideal of $\mathfrak{h}$, while $\mathfrak{S}^{\sharp}$ and $\mathfrak{S}$ are certain algebras of singular integral operators over the boundary $\boldsymbol{R}^{n+1}$.

Therefore to each operator $E \in \mathbb{E} \sharp$ (or $(E)$ there can be associated an operator valued symbol $\tau_{E}(m) \in \mathbb{C}(\mathfrak{h})$ such that $1+E$ is Fredholm if and only if $1+\tau_{E}(m)$ is regular for all $m$. The construction of a Fredholm inverse for $A \in \mathfrak{A}$ will therefore depend on two symbols: first we invert the operator modulo $\mathbb{E}$, if the complex-valued symbol

${ }^{1}$ Supported by contract AF-AFOSR 553-64. 
$\sigma_{\boldsymbol{A}}$ does not vanish; then we invert an operator $1+E \bmod \mathfrak{k}$, which depends on another, operator-valued symbol.

2. It is well known that the operators (1) have representations as (regular or singular) integral operators. Specifically

$$
\Lambda_{d}=\Lambda_{-}-\Lambda_{+}, \quad \Lambda_{n}=\Lambda_{-}+\Lambda_{+}
$$

with

(2) $\quad \Lambda_{ \pm} u=(2 / \pi)^{1 / 2}(2 \pi)^{-(n+1) / 2} \int_{R_{+}^{n+1}} K_{n / 2}\left(t_{ \pm}\right) t_{ \pm}^{-n / 2} u\left(x^{\prime}, y^{\prime}\right) d x^{\prime} d y^{\prime}$

and

$$
t_{ \pm}=\left(\left|x-x^{\prime}\right|^{2}+\left|y \pm y^{\prime}\right|^{2}\right)^{1 / 2}
$$

where $K_{\nu}(s)$ denotes the modified Bessel function as in MagnusOberhettinger [6, p. 28]. All other operators (1) experience similar decompositions and we therefore may generate $\mathfrak{A}^{*}$ by the following operators as well, which are integral operators:

(4) $\Lambda_{ \pm}, S_{ \pm}=-i \partial / \partial y \Lambda_{ \pm}, \quad S_{j, \pm}=-i \partial / \partial x_{j} \Lambda_{ \pm}, \quad j=1, \cdots, n$.

Note that

$$
\begin{aligned}
S_{ \pm} u= & i(2 / \pi)^{1 / 2}(2 \pi)^{-(n+1) / 2} \\
& \cdot \int_{R_{+}^{n+1}} K_{n / 2+1}\left(t_{ \pm}\right)\left(y \pm y^{\prime}\right) / t_{ \pm}^{n / 2+1} u\left(x^{\prime}, y^{\prime}\right) d x^{\prime} d y^{\prime}
\end{aligned}
$$

and

$$
\begin{aligned}
S_{j, \pm} u= & i(2 / \pi)^{1 / 2}(2 \pi)^{-(n+1) / 2} \\
& \cdot \int_{R_{+}^{n+1}} K_{n / 2+1}\left(t_{ \pm}\right)\left(x_{j}-x_{j}^{\prime}\right) / t_{ \pm}^{n / 2+1} u\left(x^{\prime}, y^{\prime}\right) d x^{\prime} d y^{\prime} .
\end{aligned}
$$

3. Let $F$ denote the unitary operator of $\mathfrak{S}$ induced by the Fourier transform, with respect to the $x$-variable only:

(7) $\quad F u(x, y)=(2 \pi)^{-n / 2} \int_{R^{n}} e^{-i x \cdot \xi} u(\xi, y) d \xi$ for $u \in C_{0}^{\infty}\left(R_{+}^{n+1}\right)$

and let the unitary operator $T$ be defined by

$$
(T u)(x, y)=\sigma^{-1 / 2} u(x, y / \sigma), \quad\left(T^{-1} u\right)(x, y)=\sigma^{1 / 2} u(x, y \sigma)
$$


with $\sigma=\left(1+|x|^{2}\right)^{1 / 2}$. Let $U=T F$; then we find that

(9) $U S_{ \pm} U^{-1}=P_{ \pm}, \quad U S_{j, \pm} U^{-1}=x_{j} / \sigma Q_{ \pm}, \quad U \Lambda_{ \pm} U^{-1}=1 / \sigma Q_{ \pm}$

with

$$
P_{ \pm} u=i / \pi \int_{0}^{\infty} K_{1}\left(\left|y \pm y^{\prime}\right|\right) \operatorname{sgn}\left(y \pm y^{\prime}\right) u\left(x, y^{\prime}\right) d y^{\prime}
$$

and

$$
Q_{ \pm} u=1 / \pi \int_{0}^{\infty} K_{0}\left(\left|y \pm y^{\prime}\right|\right) u\left(x, y^{\prime}\right) d y^{\prime}
$$

with sgn $t=0, \pm 1$ if $t=0,>0,<0$, resp.

Let $\mathfrak{h}=\mathscr{L}^{2}\left(R_{+}^{1}\right)$ and, for a moment, let $D_{d}$ and $D_{n}$ denote the operators $\Delta_{d}$ and $\Delta_{n}$ as introduced initially, but for $n=0$. Then we see at once that we may reinterpret $P_{ \pm}, Q_{ \pm}$above as operators on $\mathfrak{h}$ and that then

$$
\begin{gathered}
\left(1-D_{d}\right)^{-1 / 2}=Q_{-}-Q_{+}, \quad\left(1-D_{n}\right)^{-1 / 2}=Q_{-}+Q_{+} \\
-i \partial / \partial y\left(1-D_{d}\right)^{-1 / 2}=P_{-}-P_{+}, \quad-i \partial / \partial y\left(1-D_{n}\right)^{-1 / 2}=P_{-}+P_{+}
\end{gathered}
$$

while we get

$$
\mathfrak{Q}=\mathfrak{l} \otimes \mathfrak{h}, \quad \mathfrak{l}=\mathfrak{L}^{2}\left(R^{n}\right)
$$

and the relations (9) take the form

$$
\begin{gathered}
U S_{ \pm} U^{-1}=I \otimes P_{ \pm}, \quad U S_{j, \pm} U^{-1}=\left(x_{j} / \sigma\right) \otimes Q_{ \pm}, \\
U \Lambda_{ \pm} U^{-1}=(1 / \sigma) \otimes Q_{ \pm} .
\end{gathered}
$$

In (13) $\hat{\otimes}$ denotes the topological tensor product.

4. We notice that the operators $P_{ \pm}, Q_{ \pm}$of $\$ 1$ are evidently in the algebra $\mathfrak{F}$ as introduced in $[4, \S 5]$. In particular, $Q_{+}$is a compact operator of $\mathfrak{h}, Q_{-}$is an even Wiener-Hopf convolution with $\mathcal{L}^{1}$-kernel, and $P_{ \pm}$differ from $c K_{ \pm}^{0}$, with the operators $K_{ \pm}^{0}$ as in [4] and a suitable constant $c$, only by a compact operator each. It is also easily seen that $\mathfrak{F}$ may be generated as a $C^{*}$-algebra with unit by $\mathfrak{C}(\mathfrak{h}), P_{ \pm}, Q_{ \pm}$as well as by the generators listed in [4].

Definition. (a) (\$\& denotes the $C^{*}$-subalgebra of $\mathfrak{L}(\$)$ without unit generated by the operators of the form

$$
U^{*}(a(x) \otimes C) U
$$

with $a(x) \in \mathfrak{C}\left(B^{n}\right), C \in \mathbb{E}(\mathfrak{h})$. 
(b) $\mathfrak{A}^{\#}$ denotes the $C^{*}$-algebra with unit generated by $\&+$ above and all operators $S_{ \pm}, S_{j, \pm}, \Lambda_{ \pm}, j=1, \cdots, n$.

Note. As in [5] $B^{n}$ denotes the smallest compactification of $R^{n}$ into which the mapping $\rho: R^{n} \rightarrow\{|x|<1\}$ defined by

$$
\rho(x)=(2 / \pi) x /|x| \arctan |x|, x \neq 0, \quad=0 \text { for } x=0
$$

can be continuously extended.

We then have

THEOREM 1. (5* is a closed two-sided ideal of the $C^{*}$-algebra $\mathfrak{2}^{\#}$. The algebra $\mathfrak{U}^{\#} / \mathfrak{S}^{\#}$ is commutative and isometrically isomorphic to the function algebra $\mathfrak{C}\left(\mathfrak{T T}^{\#}\right)$ with the compact Hausdorff space $\mathfrak{T} \mathbf{C}^{\#}$ obtained from the product $B^{n} \times \mathfrak{T}(\mathfrak{F})$ by identifying all points of $B^{n}$ over each point of the straight line segment $x=0,-\infty<t<+\infty, \xi=\infty$ in the space $\mathfrak{T}(\mathfrak{F})$ as defined in $[4,85]$.

Clearly does not contain compact operators, except 0 . On the other hand, $\mathfrak{S}^{*}$ is contained in the $R$-algebra $U^{*}(\mathfrak{S}(\mathfrak{f}) \hat{\otimes} \mathfrak{L}(\mathfrak{h})) U=\mathfrak{\Im}$ and Theorem 1 relates the $\Im$-Fredholm property of $A \in \mathfrak{A}$ to the nonvanishing of a continuous function over $\mathfrak{T \#}$. (See [2], [3].)

Note that $M^{\#}$ is homeomorphic to an $n+1$-ball $B^{n+1}$ with the endpoints of a one-dimensional interval $I^{1}$ attached to it at two distinguished points.

5. Let $H^{n+1}$ denote the closure of $R_{+}^{n+1}$ in $B^{n+1}$. It then is an easy consequence of results published in [5] that the commutators $\left[S_{ \pm}, b\right],\left[S_{j, \pm}, b\right],\left[\Lambda_{ \pm}, b\right], j=1, \cdots, n$ are all in $\mathfrak{E}(\mathfrak{W})$, for $b \in \mathcal{C}\left(\boldsymbol{H}^{n+1}\right)$.

Definition. (a) $\&$ denotes the $C^{*}$-algebra without unit generated by $\left(\mathfrak{S}\left(\mathfrak{S )}\right.\right.$ and all products $b E, E b, b \in C\left(H^{n+1}\right), E \in(\$ *$.

(b) $\mathfrak{A}$ denotes the $C^{*}$-algebra with unit generated by $\mathfrak{S}(\mathfrak{S})$, $\mathfrak{U}^{*}$ and $C\left(\boldsymbol{H}^{n+1}\right)$.

We then have the following main result.

ThEOREM 2. (a) $\mathfrak{E} \subset \mathfrak{A}$ is a closed two-sided ideal of $\mathfrak{A}$, and $\mathfrak{X} / \mathfrak{E}$ is commutative.

(b) $\mathfrak{S}=\mathbb{S}(\mathfrak{S})$ is a closed two-sided ideal of $E$.

(c) The Gelfand space $\mathfrak{T}$ of $\mathfrak{A} / \mathfrak{E}$ is (homeomorphic to) the following subset of the cartesian product $\mathfrak{T I}^{\#} \times H^{n+1}$ (शTt as in Theorem 1):

(i) Over the boundary at $y=\infty$ of $H^{n+1}$ one gets all points of $B^{n+1} \subset \mathbb{N}^{*}$.

(ii) Over interior points of $R_{+}^{n+1} \subset H^{n+1}$ one gets the boundary $\partial B^{n+1}$ of the ball $B^{n+1} \subset$ Irt.

(iii) Over the boundary $y=0$ of $R_{+}^{n+1} \subset H^{n+1}$ one gets the interval $I^{1}$ and the boundary $\partial B^{n+1}$ of the ball $B^{n+1} \subset H^{n+1}$. 

Trt.

(iv) Over the points $y=0,|x|=\infty$ of $H^{n+1}$ one gets the whole space

(d) The algebra $\mathbb{E} / \mathfrak{C}$ is isometrically isomorphic to the algebra $\mathfrak{e}\left(\mathfrak{T H}_{1}, \mathfrak{C}(\mathfrak{h})\right)$ of all continuous functions from a compact Hausdorff-space $\mathfrak{T}_{1}$ to the compact ideal $\mathfrak{S}(\mathfrak{h})$ of the Hilbert-space $\mathfrak{h}$.

(e) The space $\mathfrak{T}_{1}$ is (homeomorphic to) the set

$$
\partial B^{n} \times B^{n} \cup B^{n} \times \partial B^{n} \subset B^{n} \times B^{n},
$$

(i.e., topologically is a $2 n-1$ sphere).

Definition. (a) To any $A \in \mathfrak{Y}$ we associate $\sigma_{A} \in \mathcal{C}(\mathfrak{T})$ defined as the function associated to the coset of $A \bmod \&$ by the Gelfand isomorphism of $\mathfrak{A} / \mathbb{E} . \sigma_{A}$ will be called the $\mathbb{E}$-symbol of $A \in \mathfrak{A}$.

(b) To any $E \in \mathbb{E}$ we associate $\tau_{E} \in \mathfrak{C}\left(\mathfrak{T C}_{1}, \mathfrak{C}(\mathfrak{h})\right)$ defined as image of the coset of $E \bmod \mathbb{E}(\$)$ under the isomorphism (d) of Theorem 2. $\tau_{E}$ will be called the (S-symbol of $E \in \mathbb{E}$.

Theorem 3. (a) $A$ necessary condition for $A \in \mathfrak{A}$ to be Fredholm is that its (5-symbol does never vanish on $\mathfrak{T}$.

(b) $A \in \mathfrak{U}$ with $\sigma_{A} \neq 0$ on $\mathfrak{T}$ possesses an inverse $B \in \mathfrak{U} \bmod (\mathbb{E}$ such that $1-A B, 1-B A \in \mathbb{E}$.

(c) $A \in \mathfrak{A}$ with $\sigma_{A} \neq 0$ is Fredholm if and only if for some $($-inverse $B$ of $A$ we have

$$
\left(1+\tau_{(A B-1)}(m)\right) \text { a regular operator of } \mathfrak{R}(\mathfrak{h})
$$

for all $m \in \mathfrak{M T}_{1}$.

6. The proof of Theorem 2 rests on the following facts partly of independent interest.

THEOREM 4. If $b \in C\left(H^{n+1}\right)$ vanishes on the boundary $y=0$ then $E b$, $b E$ are compact, for all $E \in \&^{\sharp}$.

The result of Theorem 4 may be expressed by saying that all operators of $₹$ are "compact, except over the boundary of $R^{n+1}$."

THEOREM 5. We have $U \mathbb{E} U^{*}=\mathfrak{S} \hat{\otimes} \mathfrak{S}(\mathfrak{h})$ with the algebra $\mathfrak{S}$ as in $[\mathbf{5}$, appendix].

This completely clarifies the structure of the ideal $\&$ and assertions (d) and (e) of Theorem 2 become evident, in view of [5] and [1].

While the proof of assertions (a) and (b) is a verification only, one may employ techniques as in [5] to obtain the precise extent of the space $\mathfrak{T}$.

We notice that the operators of our algebra $\mathfrak{A}$ are similar to those considered by Vishik and Eskin [7], for instance. 
Applicability of our results should strongly depend on the explicit construction of inverses mod $\&$ and of Fredholm inverses.

Especially we also expect results concerning pseudo-differential operators involving boundary conditions in a half-space like those in $[4, \S 6]$.

\section{REFERENCES}

1. M. Breuer and $\mathrm{H}$. O. Cordes, On Banach algebras with $\sigma$-symbol, Part II, J. Math. Mech. 14 (1965), 299-314.

2. H. O. Cordes, On a class of $C^{*}$-algebras, Math. Ann. 170 (1967), 283-313.

3. - Über eine nichtalgebraische Characterisierung von 3 -Fredholmoperatoren, Math. Ann. 163 (1966), 212-229.

4. - Pseudo-differential operators on half-line, J. Math. Mech. (to appear).

5. H. O. Cordes and E. Herman, Remarks on the Gel'fand theory of pseudo-differential operators, Amer. J. Math. (to appear).

6. W. Magnus and F. Oberhettinger, Formeln und Sätze für die speziellen Funktionen der Mathematischen Physik, Springer, Berlin, 1948.

7. M. I. Vishik and G. I. Eskin, Equations in convolutions in a bounded domain, Uspehi Mat. Nauk 20 (1965), 90-152=Russian Math. Surveys 20 (1965), 85-151.

University of California, Berkeley, California 94720 